\title{
VRK2 gene expression in schizophrenia, bipolar disorder and healthy controls
}

Martin Tesli,* Katrine Verena Wirgenes,* Timothy Hughes, Francesco Bettella, Lavinia Athanasiu, Eva S. Hoseth, Mari Nerhus, Trine V. Lagerberg, Nils E. Steen, Ingrid Agartz, Ingrid Melle, Ingrid Dieset, Srdjan Djurovic and Ole A. Andreassen

\section{Background}

Common variants in the Vaccinia-related kinase 2 (VRK2) gene have been associated with schizophrenia, but the relevance of its encoded protein VRK2 in the disorder remains unclear.

\section{Aims}

To identify potential differences in VRK2 gene expression levels between schizophrenia, bipolar disorder, psychosis not otherwise specified (PNOS) and healthy controls.

\section{Method}

VRK2 mRNA level was measured in whole blood in 652 individuals (schizophrenia, $n=201$; bipolar disorder, $n=167$; PNOS, $n=61$; healthy controls, $n=223$ ), and compared across diagnostic categories and subcategories. Additionally, we analysed for association between 1566 VRK2 single nucleotide polymorphisms and mRNA levels.

\section{Results}

We found lower VRK2 mRNA levels in schizophrenia compared with healthy controls $\left(P<10^{-12}\right)$, bipolar disorder
$\left(P<10^{-12}\right)$ and PNOS $(P=0.0011)$, and lower levels in PNOS than in healthy controls $(P=0.0042)$ and bipolar disorder $(P=0.00026)$. Expression quantitative trait loci in close proximity to the transcription start site of the short isoforms of the VRK2 gene were identified.

\section{Conclusions}

Altered VRK2 gene expression seems specific for schizophrenia and PNOS, which is in accordance with findings from genome-wide association studies. These results suggest that reduced VRK2 mRNA levels are involved in the underlying mechanisms in schizophrenia spectrum disorders.

\section{Declaration of interest}

M.T. received speaker's honorarium from Medivir. O.A.A. received speaker's honoraria from GlaxoSmithKline, Lundbeck and Otsuka.

\section{Copyright and usage}

(c) The Royal College of Psychiatrists 2016.
Schizophrenia is a severe psychiatric disorder with high heritability estimates $^{1,2}$ and polygenic inheritance. ${ }^{3}$ Despite the identification of common genetic variants through large genome-wide association studies (GWAS), little is known about the relation between DNA variants, gene expression and the clinical manifestations of the disorder. We have previously demonstrated that expression levels of candidate genes identified through GWAS, such as NOTCH4, TCF4 and ANK3, are significantly altered in schizophrenia and bipolar disorder, ${ }^{4-6}$ thus providing evidence that mRNA levels in blood might be a useful marker for further exploration of underlying disease mechanisms. Among the most consistently reported schizophrenia risk genetic variants are single nucleotide polymorphisms (SNPs) in vaccinia-related kinase 2 (VRK2). ${ }^{3,7,8}$ Although one family study implicated a rare VRK2 variant in bipolar disorder, ${ }^{9}$ stronger associations have been found in schizophrenia., ${ }^{3,10}$ The VRK2 gene (chromosome 2p16) encodes a protein in the VRK family of serine/threonine protein kinases, first identified in highly proliferative cells, such as testis, thymus and fetal liver, ${ }^{11}$ and later shown to be expressed in human brain, through all stages of life (http://braincloud.jhmi.edu/). The VRK2 protein has been found to be involved in signaling pathways regulating apoptosis, tumor cell growth ${ }^{12}$ and the immune response, ${ }^{13}$ and could be relevant for neurological diseases. ${ }^{14}$ In particular, variants in VRK2 have been associated with epilepsy. ${ }^{15}$ These findings are of interest in the light of the associations found between immune-related genes in the major histocompatibility complex region at chromosome 6 and schizophrenia, ${ }^{3}$ and the polygenic overlap between schizophrenia and neurological

*These authors contributed equally to this work. disorders. ${ }^{16}$ However, the relation between identified VRK2 schizophrenia risk SNPs, VRK2 mRNA level and the clinical phenotype remains to be elucidated. One study reported altered $V R K 2$ expression in blood cells of patients with schizophrenia $(n=20)$ compared with healthy controls $(n=18)$, but the result was only trend significant and no expression quantitative trait loci (eQTL) were identified. ${ }^{7}$

We aimed to identify any abnormal VRK2 mRNA blood levels in schizophrenia compared with healthy controls. Further, we wanted to determine potential diagnostic specificity by comparing schizophrenia with psychosis not otherwise specified (PNOS) and bipolar disorder, as PNOS can be regarded as a less severe schizophrenia spectrum disorder ${ }^{17,18}$ and bipolar disorder shares clinical features and genetic risk with schizophrenia. ${ }^{18-20}$ Additionally, we performed association analyses between SNPs in the VRK2 region and mRNA level, to identify cis-acting eQTLs.

\section{Method}

\section{Sample characteristics}

Our sample consisted of 652 individuals, organised into the diagnostic categories schizophrenia, bipolar disorder, PNOS and healthy controls. The schizophrenia category $(n=201)$ (SZ group) included the subcategories schizophrenia $(n=158)$, schizophreniform disorder $(n=17)$ and schizoaffective disorder $(n=26)$. The bipolar disorder category $(n=167)$ (BD group) included bipolar disorder type $1(n=103)$, bipolar disorder type $2(n=51)$ and bipolar disorder not otherwise specified (BDNOS) $(n=13)$. The PNOS category included 61 individuals, and 223 participants were included in the healthy control category (CTR group). The 
patients were diagnosed according to the Structured Clinical Interview for DSM-III-R (SCID $\left.{ }^{21}\right)$. Overall, 78 patients $(75.7 \%)$ with bipolar disorder type 1, 11 patients $(21.6 \%)$ with bipolar disorder type 2 and 8 patients $(61.5 \%)$ with BDNOS had experienced at least one Structural Clinical Interview for DSM-III-R SCID-verified psychotic episode. Healthy controls were randomly recruited from the same catchment area as the patients, and underwent an interview where demographic and clinical information was obtained. Our sample consisted of White Northern European participants (mainly Norwegians). We have previously demonstrated our sample to be genetically homogeneous, based on linkage clustering as well as self-reports on ancestry. ${ }^{6,22,23}$ Clinical evaluation of the patients and healthy controls participating in this study is described in detail in previous reports. ${ }^{6}$ Demographical data are shown in Table 1.

The Norwegian Scientific Ethical Committees and the Norwegian Data Protection Agency approved the study. All participants have given written informed consent prior to inclusion into the project.

\section{RNA measurement}

Blood samples were collected using Tempus Blood RNA Tubes (Life Technologies Corporation, Carlsbad, California, USA). Total RNA was extracted with ABI PRISM 6100 Nucleic Acid PrepStation (Life Technologies Corporation, Carlsbad, California, USA) and TEMPUS 12-port RNA Isolation Kit according to the manufacturer's protocol. High-Capacity cDNA Reverse Transcription Kit (Life Technologies Corporation, Carlsbad, California, USA) was used for reverse transcription of $1 \mu \mathrm{g}$ RNA. Quantitative reverse transcriptase polymerase chain reaction was performed on ABI PRISM 7900HT Sequence Detection System by using TaqMan Gene Expression Assays (VRK2: Hs00241738_m1; Life Technologies Corporation, Carlsbad, California, USA) and the gene ACTB (Hs99999903_m1; Life Technologies Corporation, Carlsbad, California, USA) was used as endogenous control. We analysed the expression stability of 16 endogenous control genes in 16 samples (4 healthy controls, 4 bipolar disorder, 4 schizophrenia and 4 PNOS) using the geNorm method. ${ }^{24}$ We found that all tested endogenous controls to be below the recommend $\mathrm{M}$-value cutoff of 0.5 and ACTB is one of the most stable of these (see Fig. DS1 in the online supplement). The instruction protocol from the manufacturer was strictly followed. The results were processed in RQ Manager 1.2.1. VRK2 mRNA ÄCt (cycling threshold) in each individual was calibrated against mean VRK2 mRNA $\Delta$ Ct in the CTR group. The following formula was used for statistical analyses in this report: $2^{(-\Delta \Delta \mathrm{Ct})}$, as described previously. ${ }^{6}$

\section{Genotyping and SNP selection}

The sample was genotyped at Expression Analysis (Durham, North Carolina, USA) using the Affymetrix Genome-Wide Human SNP array 6.0 (Affymetrix, Santa Clara, California, USA). Quality control was performed using PLINK (version 1.07; http://pngu.mgh.harvard.edu/purcell/plink/). ${ }^{25}$ Overall, significant plate-specific markers were set to missing (in that plate). One of two duplicates, one of two relatives (identity by descent $>0.1875$ ), samples with a reported gender differing from that determined by $\mathrm{X}$ chromosome marker homozygosity, mixupsamples (as calculated by pairwise genome-wide identity by state), samples with non-European ancestry (as calculated with HapMap3 (Wellcome Trust Sanger Institute, Cambridge, UK) and multidimensional scaling) and samples with individual genotyping below $95 \%$, were excluded. SNPs were excluded if they had minor allele frequency $<1 \%$, low yield $(<95 \%)$ or deviated from Hardy-Weinberg equilibrium $(P<0.001)$.

\section{MACH imputed data}

Candidate SNPs were imputed with $\mathrm{MACH}^{26}$ using the European samples available in the Phase I release of the 1000 Genomes Project (www.sph.umich.edu/csg/abecasis/MACH/download/ 1000G-PhaseI-Interim.html) after the quality control described above. In addition, all SNPs not present in the 1000 Genomes reference, as well as all SNPs where strand alignment was ambiguous (A/T and G/C SNPs), were removed from the sample data-sets. Imputations were carried out in a three-stage process using the ChunkChromosome (http://genome.sph.umich.edu/ wiki/ChunkChromosome), MACH (www.sph.umich.edu/csg/ abecasis/MaCH/download/) and minimac programs (http:// genome.sph.umich.edu/wiki/Minimac). First, the data-sets were broken into 2500 SNP pieces, with 500 SNP overlap using ChunkChromosome. Second, each piece was phased using MACH (40 rounds, 400 states). Third, each phased piece was imputed to the 1000 Genomes European reference panel using minimac (20 rounds, 400 states). Minimac provides an estimated $r^{2}$ score that provides a quality metric for each imputed SNP. All SNPs with $r^{2}<0.5$ were excluded from further analysis leaving 9584802 SNPs. 1566 imputed VRK2 SNPs based on UCSC (hg 19) Chr2: $57,900-58,600$ were extracted for inclusion in the current analyses. This region was selected to cover all risk SNPs from the Psychiatric Genomics Consortium schizophrenia case-control study ${ }^{3}$ in and around the VRK2 gene (based on summary statistics from www.broadinstitute.org/mpg/ricopili/).

\section{Statistical analyses}

\section{VRK2 mRNA across diagnostic categories}

Using IBM's SPSS software package for Windows, version 21, differences in VRK2 mRNA levels between the diagnostic categories (bipolar disorder, schizophrenia, PNOS and healthy controls) were investigated with an ANOVA model, followed by pairwise comparisons adjusted with the Tukey method. Seven individuals were removed owing to deviant VRK2 mRNA levels defined as 3 standard deviations from the mean. Post-hoc analyses were performed with age, gender, alcohol use (Alcohol Use Disorders Identification Test, AUDIT) ${ }^{27}$ and illegal drug use (Drug Use Disorders Identification Test, DUDIT ${ }^{28}$ as covariates in an ANCOVA model in the total sample. Additionally, medication status (dichotomised because of non-normal distribution) for antipsychotics, antidepressants, anticonvulsants, lithium, hypnotics and psychostimulants were regressed against VRK2 mRNA level within the patients in a multiple hierarchical regression model with diagnostic category, gender and age as covariates. The same procedure was undertaken for daily use of nicotine (yes/no), as this information was not available in the CTR group. Effect sizes (Nagelkerke $R^{2}$ ) for the significant differences in mRNA level for pairwise comparisons between the BD, SZ, PNOS and CTR groups were estimated with logistic regressions. To assess the consistency of the findings, ANOVA analyses were undertaken in the diagnostic subcategories, as well as in males and females separately for the main diagnostic categories. Additionally, potential difference in VRK2 mRNA level between bipolar disorder with psychotic symptoms and bipolar disorder without psychotic symptoms was assessed with a $t$-test.

\section{Genetic associations with VRK2 mRNA levels}

Association analyses between 1566 imputed VRK2 SNPs and mRNA levels were performed with a linear regression model in PLINK (version 1.07; http://pngu.mgh.harvard.edu/purcell/ plink/). ${ }^{25}$ These analyses were conducted in the total sample and in the $\mathrm{BD}, \mathrm{SZ}, \mathrm{PNOS}$ and CTR groups separately, with gender 
Table 1 Demographics and clinical characteristics

\begin{tabular}{|c|c|c|c|c|c|c|c|}
\hline & \multirow[b]{2}{*}{$\begin{array}{l}\text { SZ group } \\
(n=201)\end{array}$} & \multirow[b]{2}{*}{$\begin{array}{l}\text { BD group }{ }^{a} \\
(n=167)\end{array}$} & \multirow[b]{2}{*}{$\begin{array}{l}\text { PNOS group } \\
\quad(n=61)\end{array}$} & \multirow[b]{2}{*}{$\begin{array}{l}\text { CTR group } \\
(n=223)\end{array}$} & \multicolumn{3}{|c|}{ ANOVA $\chi^{2}$ analysis } \\
\hline & & & & & $F / \chi^{2}$ & $P$ & Post-hoc \\
\hline Female, \% & 38.3 & 62.9 & 39.3 & 44.8 & $\chi^{2}=24.8$ & $<0.001$ & - \\
\hline Age, years: mean (s.d.) & $30.9(9.6)$ & $34.3(11.5)$ & $32.8(10.3)$ & $33.1(9.3)$ & $F=3.3$ & 0.020 & $(\mathrm{BD}>\mathrm{SZ})$ \\
\hline Age at onset, years: mean (s.d.) & $23.3(8.3)$ & $23.6(10.1)$ & $27.2(10.2)$ & - & $F=3.8$ & 0.022 & (PNOS > BD,SZ) \\
\hline GAF symptom, mean (s.d.) & $40.8(11.0)$ & $55.7(11.3)$ & $50.0(14.1)$ & - & $F=-72.0$ & $<0.001$ & $(\mathrm{BD}>\mathrm{PNOS}>\mathrm{SZ})$ \\
\hline GAF function, mean (s.d.) & $41.9(9.9)$ & $53.2(12.4)$ & $52.6(14.9)$ & - & $F=-44.3$ & $<0.001$ & $(\mathrm{BD}, \mathrm{PNOS}>\mathrm{SZ})$ \\
\hline PANSS positive, mean (s.d.) & $15.6(5.4)$ & $10.0(3.6)$ & $12.7(4.1)$ & - & $F=63.6$ & $<0.001$ & $(\mathrm{SZ}>\mathrm{PNOS}>\mathrm{BD})$ \\
\hline PANSS negative, mean (s.d.) & $16.1(6.2)$ & $10.5(3.7)$ & $11.7(5.1)$ & - & $F=51.0$ & $<0.001$ & $(\mathrm{SZ}>\mathrm{PNOS}, \mathrm{BD})$ \\
\hline PANSS total, mean (s.d.) & $65.0(16.8)$ & $46.4(10.9)$ & 53.7 (14.5) & - & $F=71.6$ & $<0.001$ & $(\mathrm{SZ}>\mathrm{PNOS}>\mathrm{BD})$ \\
\hline IDS, mean (s.d.) & 17.5 (11.9) & $17.4(12.8)$ & $15.2(11.4)$ & - & - & - & - \\
\hline YMRS, mean (s.d.) & $6.1(5.1)$ & $3.0(4.0)$ & $4.0(4.1)$ & - & $F=19.1$ & $<0.001$ & (SZ>PNOS,BD) \\
\hline Nicotine, ${ }^{b} \%$ & 57.9 & 58.8 & 54.4 & - & - & - & - \\
\hline AUDIT, ${ }^{c}$ mean (s.d.) & $6.4(6.6)$ & 7.7 (6.3) & $8.2(7.9)$ & $5.4(3.0)$ & $F=5.1$ & 0.002 & (PNOS,BD > SZ,CTR) \\
\hline DUDIT, ${ }^{d}$ mean (s.d) & $2.5(5.9)$ & $2.5(6.3)$ & $4.7(8.0)$ & $0.2(0.8)$ & $F=11.0$ & $<0.001$ & (PNOS,SZ,BD > CTR) \\
\hline \multicolumn{8}{|l|}{ Medication status, \% } \\
\hline Medicated & 90.7 & 83.9 & 78.9 & - & $F=3.2$ & 0.040 & - \\
\hline Antipsychotics & 77.1 & 49.7 & 67.2 & - & $F=16.2$ & $<0.001$ & (SZ,PNOS > BD) \\
\hline Lithium & 2.5 & 14.4 & 0.0 & - & $F=13.5$ & $<0.001$ & (BD > SZ,PNOS) \\
\hline Anticonvulsants & 17.4 & 42.5 & 9.8 & - & $F=21.6$ & $<0.001$ & (BD > SZ,PNOS) \\
\hline Antidepressants & 25.4 & 38.3 & 34.4 & - & $F=3.7$ & 0.026 & $(\mathrm{BD}>\mathrm{SZ})$ \\
\hline Hypnotics & 14.4 & 12.0 & 4.9 & - & - & - & - \\
\hline Psychostimulants & 0.0 & 1.2 & 1.6 & - & - & - & - \\
\hline \multicolumn{8}{|c|}{$\begin{array}{l}\text { SZ, schizophrenia; BD, bipolar disorder; PNOS, psychotic disorder not otherwise specified; CTR, healthy controls; PANSS, Positive and Negative Syndrome Scale; }{ }^{29} \text { IDS, Inventory of } \\
\text { Depressive Symptomatology; }{ }^{30} \mathrm{GAF}, \text { Global Assessment of Functioning; }{ }^{31} \text { YMRS, Young Mania Rating Scale; } 32 \text { AUDIT, Alcohol Use Disorders Identification Test; DUDIT, Drug Use } \\
\text { Disorders Identification Test. } \\
\text { a. } n=103 \text { bipolar disorder type I (61.7\%); } n=51 \text { bipolar disorder type II (30.6\%) and } n=13 \text { bipolar disorder not otherwise specified (7.8\%). } \\
\text { b. Daily use of nicotine. } \\
\text { c. Past } 12 \text { months, problematic use defined as score } \geqslant 8 \text { in males and } \geqslant 7 \text { in females. } \\
\text { d. Past } 12 \text { months, problematic use defined as score } \geqslant 6 \text { in males. }\end{array}$} \\
\hline
\end{tabular}

and age as covariates, as well as diagnostic category in the total sample. Gene-wide significance threshold for association between 1566 VRK2 SNPs and VRK2 mRNA was computed with the aid of matSpD ${ }^{33}$ taking into account the SNP correlations reflected by the sample's linkage disequilibrium structure in the region of interest. The effective number $(n=174)$ of independent tests was computed using Li's procedure. Total sample size for these analyses after quality control procedures was 575 (schizophrenia, $n=169$; bipolar disorder, $n=146$; PNOS, $n=50$; healthy controls, $n=210$ ).

\section{Results}

\section{VRK2 mRNA levels across diagnostic categories}

VRK2 mRNA levels differ significantly between the SZ, BD, PNOS and CTR groups (ANOVA, $F=53.6$, d.f. $=3,648, P=5.8 \times 10^{-31}$ ) (Fig. 1 and Table 2). Pairwise comparisons reveal significantly lower VRK2 mRNA levels in the SZ group than the CTR $\left(P<10^{-12}\right.$, Nagelkerke $\left.R^{2}=0.30\right)$, BD $\left(P<10^{-12}\right.$, Nagelkerke $\left.R^{2}=0.42\right)$ and PNOS groups $\left(P=0.0011\right.$, Nagelkerke $\left.R^{2}=0.13\right)$, and significantly lower levels in PNOS than healthy controls $\left(P=0.0042\right.$, Nagelkerke $\left.R^{2}=0.051\right)$ and bipolar disorder $\left(P=0.00026\right.$, Nagelkerke $\left.R^{2}=0.11\right)$. Results from the post-hoc analyses (ANCOVA) remain largely unchanged after controlling for gender, age, alcohol and drug use (online Table DS1). There are no significant effects of antipsychotics, anticonvulsants, lithium, hypnotics or psychostimulants on VRK2 mRNA level within the patients. Antidepressants are associated with lower VRK2 mRNA levels $(P=0.007)$, but this cannot explain the effect of diagnostic category on VRK2 mRNA level, as more patients with bipolar disorder than those with schizophrenia used antidepressants (Table 1). Smoking status does not influence
VRK2 mRNA levels within the patients. ANOVA analyses show a consistent pattern for the diagnostic subcategories compared with the main categories (online Fig. DS1 and Table DS2). Further, the results remain largely the same when analysed in males and females separately (online Table DS3). There were no significant differences in VRK2 mRNA level between bipolar disorder with psychotic symptoms and bipolar disorder without psychotic symptoms. In addition to significant differences in mean expression level between the diagnostic categories, the range of the distributions are only partially overlapping (Fig. 2), with no individuals with schizophrenia with relative expression value above 1.3 , whereas 45 healthy controls $(20.2 \%)$ and 25 patients with bipolar disorder (15.0\%) have such expression levels. Moreover, only 18 individuals with schizophrenia have an expression value above $1.0(9.0 \%)$, whereas 96 individuals with bipolar disorder (57.5\%) and 99 healthy controls $(44.4 \%)$ have a value above 1.0 (online Fig. DS3).

\section{Genetic associations with VRK2 mRNA levels}

$V R K 2$ SNPs in close proximity to the transcription start site of the short isoforms of the VRK2 gene are significantly associated with VRK2 mRNA levels in the total sample, with weaker effects in the $\mathrm{BD}, \mathrm{SZ}, \mathrm{PNOS}$ and CTR groups separately (online Table DS4 and Fig. 3). A distinct cluster of highly associated SNPs is located in the $25 \mathrm{~kb}$ immediately to the 5 ' of the transcription start site which is slightly closer to the transcription start site than the tag SNP (rs2312147) previously identified as significantly associated with clinical phenotype. ${ }^{8}$ Another region close to the VRK2 gene harboring three significant tag SNPs (at approximately chr2:57.9-58.0Mb), which has been previously reported, ${ }^{3}$ does not appear to be an eQTL. 


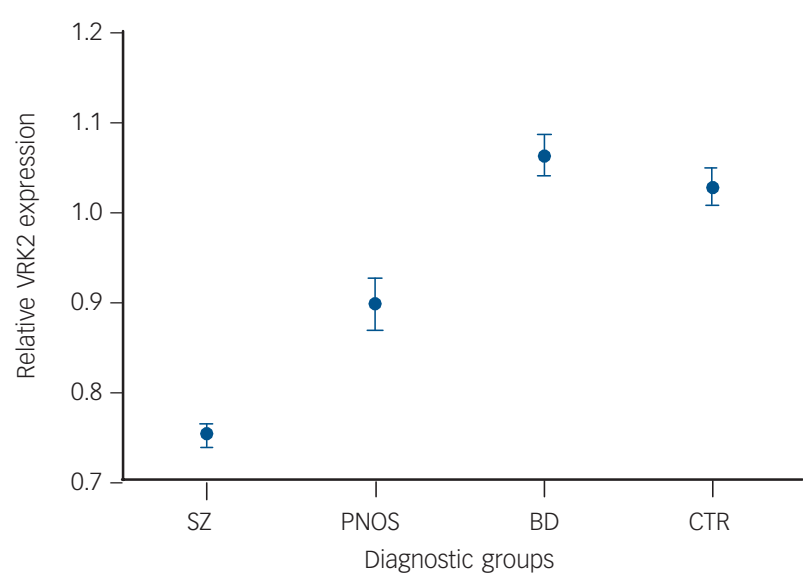

Fig. 1 VRK2 mRNA expression levels (mean value one standard error) according to diagnostic category.

VRK2 mRNA levels are significantly lower in schizophrenia (SZ) than healthy controls (CTR) $\left(P<10^{-12}\right)$, bipolar disorder (BD) $\left(P<10^{-12}\right)$ and psychosis not otherwise specified (PNOS) $(P=0.0011)$, and significantly lower in PNOS than CTR $(P=0.0042)$ and $\mathrm{BD}(P=0.00026)$.

\section{Discussion}

The main finding of the present study was a clear reduction in VRK2 mRNA levels in the SZ group compared with the CTR group. The gene expression levels in bipolar disorder were comparable to those in the CTR group, with the PNOS group intermediate between the $\mathrm{SZ}$ and $\mathrm{BD} / \mathrm{CTR}$ groups. These findings are in accordance with results from recent GWAS, where VRK2 SNPs have been more strongly associated with schizophrenia than with bipolar disorder. ${ }^{3,10}$ The finding of diagnostic specificity for reduced VRK2 mRNA levels in schizophrenia is interesting in light of recent evidence for genetic risk variants in the major histocompatibility complex region specific to schizophrenia and apparently not implicated in bipolar disorder. ${ }^{34}$ The polygenic overlap in the major histocompatibility complex region between schizophrenia and neurological disorders also seems specific to schizophrenia and not bipolar disorder. ${ }^{16}$ The intermediate gene expression levels in PNOS between the SZ and CTR groups is in

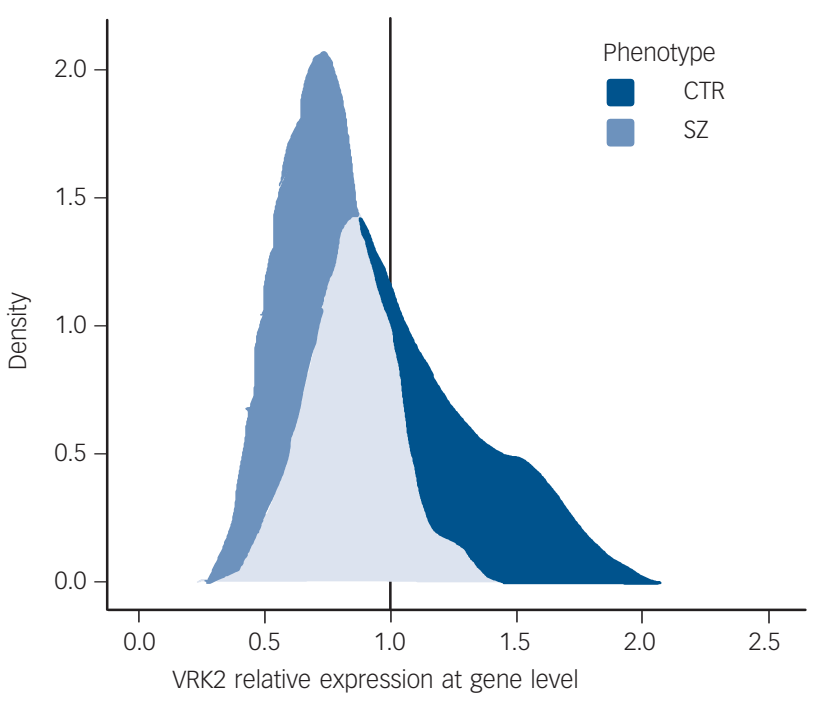

Fig. 2 Density plot for VRK2 expression in schizophrenia and healthy controls.

Smooth density estimate computed with $1 \mathrm{~d}$ kerne $\mathrm{I}^{35}$ using relative expression levels of VRK2 for the following diagnostic categories: schizophrenia (SZ) $(n=201)$ and healthy controls (CTR) $(n=223)$

line with clinical observations, as PNOS is a less severe form of psychosis than schizophrenia.

Intriguingly, VRK2 variants have been associated with epilepsy, ${ }^{36}$ and the encoded protein VRK2 has been found to be important for regulation of cell growth and inflammatory responses. ${ }^{14}$ More specifically, there is evidence that high VRK2 levels confer protection against apoptosis, ${ }^{12}$ and that VRK2 reduces the transcriptional response to interleukin- $1 \mathrm{~b},{ }^{13}$ a cytokine involved in inflammatory activity. Thus, one could speculate that reduced VRK2 levels might increase already elevated inflammatory response, in addition to inducing apoptosis, thereby inhibiting normal neurodevelopment during early stages of life, which has been suggested as an underlying mechanism in schizophrenia. ${ }^{37}$ This hypothesis is in accordance with findings of higher levels of inflammatory markers, ${ }^{38}$ brain volume

\begin{tabular}{|c|c|c|c|c|c|}
\hline \multirow[b]{2}{*}{ Diagnostic category } & \multirow[b]{2}{*}{ Mean difference } & \multirow[b]{2}{*}{ s.e. } & \multirow[b]{2}{*}{$P$} & \multicolumn{2}{|c|}{$95 \%$ confidence Interval } \\
\hline & & & & Lower limit & Upper limit \\
\hline \multicolumn{6}{|l|}{ CTR } \\
\hline SZ & 0.28 & 0.026 & $<10^{-12}$ & 0.21 & 0.34 \\
\hline $\mathrm{BD}$ & -0.034 & 0.027 & 0.60 & -0.10 & 0.036 \\
\hline PNOS & 0.13 & 0.038 & 0.0042 & 0.031 & 0.23 \\
\hline \multicolumn{6}{|l|}{ SZ } \\
\hline CTR & -0.28 & 0.026 & $<10^{-12}$ & -0.34 & -0.21 \\
\hline $\mathrm{BD}$ & -0.31 & 0.028 & $<10^{-12}$ & -0.38 & -0.24 \\
\hline PNOS & -0.15 & 0.034 & 0.0011 & -0.25 & -0.045 \\
\hline \multicolumn{6}{|l|}{$\mathrm{BD}$} \\
\hline CTR & 0.034 & 0.027 & 0.60 & -0.036 & 0.10 \\
\hline SZ & 0.31 & 0.028 & $<10^{-12}$ & 0.24 & 0.38 \\
\hline PNOS & 0.16 & 0.040 & 0.00026 & 0.061 & 0.27 \\
\hline \multicolumn{6}{|l|}{ PNOS } \\
\hline CTR & -0.13 & 0.038 & 0.0042 & -0.23 & -0.031 \\
\hline $\mathrm{SZ}$ & 0.15 & 0.039 & 0.0011 & 0.045 & 0.25 \\
\hline $\mathrm{BD}$ & -0.16 & 0.040 & 0.00026 & -0.27 & -0.061 \\
\hline
\end{tabular}


reductions ${ }^{39}$ and neurocognitive impairment in schizophrenia. ${ }^{40}$ Further, the VRK2 risk allele in rs 2312147 has been associated with reduced total brain volume and white matter volume in healthy individuals. ${ }^{7}$ The lack of association between antipsychotic medication and VRK2 mRNA levels in the current study further strengthens an argument for the role of VRK2 in the neurodevelopmental hypothesis.

The extent to which gene expression in blood can act as a proxy for brain expression remains unclear, ${ }^{41}$ however the use of blood RNA is a practical necessity if one wishes to study gene expression in a large number of individuals with psychiatric disorder and controls. Since we lack sufficient information on the nature of the blood-brain gene expression correlation, we cannot claim that what we observe in blood is an accurate reflection of expression in the brain. However, this does not detract from the fact that blood-based gene expression levels may be useful in diagnosis. ${ }^{42}$ Gene expression in blood has been found to share significant similarities with multiple central nervous system tissues, and correlation between transcripts present in both whole blood and nervous system has been estimated to be around $0.5{ }^{41}$ Further, peripheral blood was recently validated as a valuable source of information for estimation of heritability through gene expression as well as identification of eQTLs in a large twin study. ${ }^{43}$

VRK2 has nine transcripts in the RefSeq database, but these divide into two broad groups: the long isoforms with a transcription start site at approximately $58134 \mathrm{kbp}$ and the short forms with a transcription start site at approximately $58273 \mathrm{kbp}$. The expression profile of VRK2 is very similar in brain and blood, with the short form being the dominantly expressed form in both tissues. The long form is also expressed in both these tissues, but at very low levels (online Fig. DS4).

The probe used for measuring VRK2 expression in this study was chosen so as to produce an aggregate measure of expression over all RefSeq transcripts. Since we know that the short form is the most abundantly expressed, it is not surprising to observe in our data a cluster of significant SNPs near the transcription start site of the short isoforms. However, the significant SNPs from the latest Psychiatric Genomics Consortium schizophrenia study ${ }^{3}$ form a large cluster around the transcription start site of the

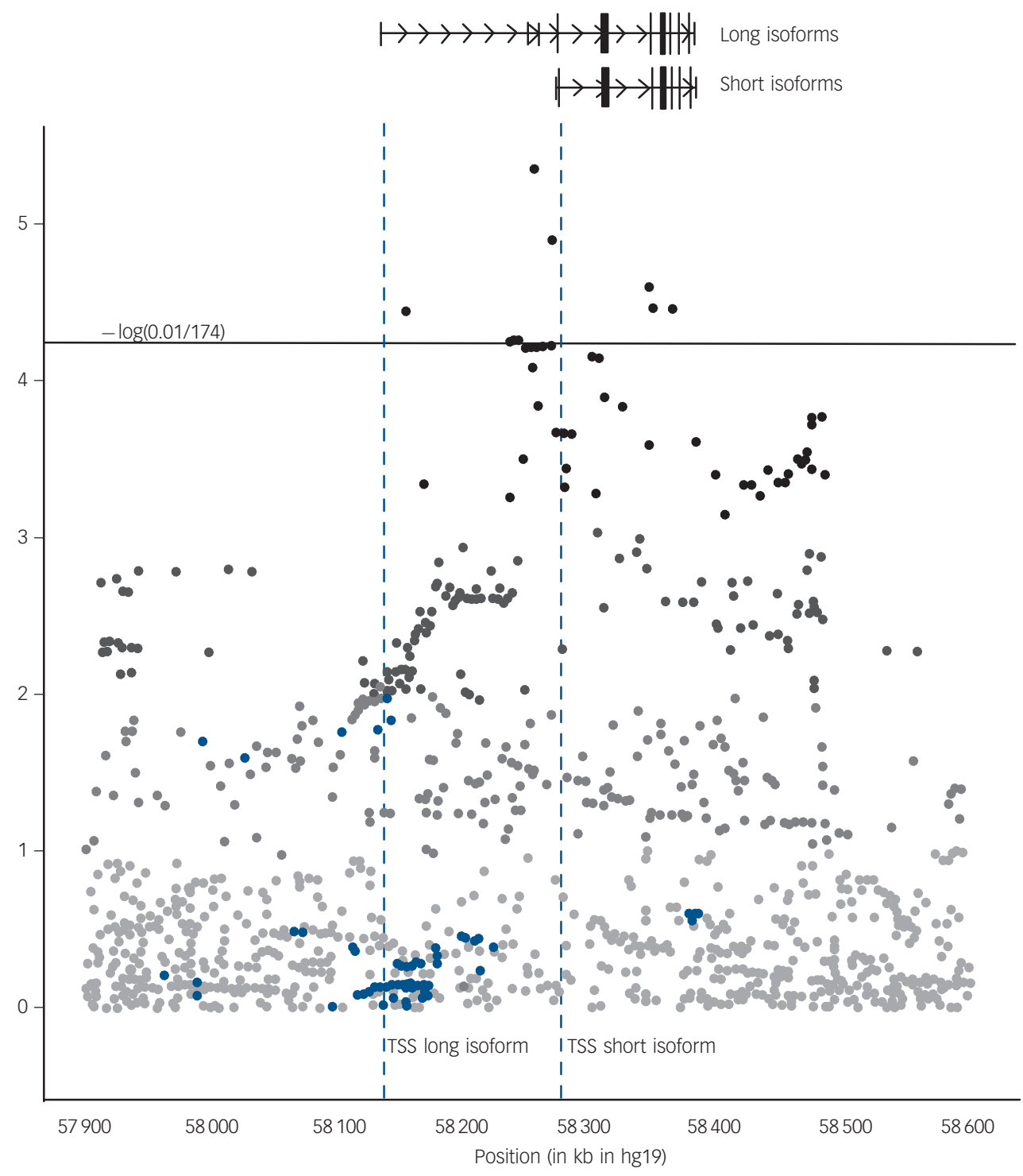

Fig. 3 VRK2 expression quantitative loci.

VRK2 expression quantitative loci identified in the total sample $(n=652)$, using a linear regression model with gender, age and diagnostic category as covariates. Blue dots: VRK2 single nucleotide polymorphisms (SNPS) included in our expression model which are genome-wide significant in the latest Psychiatric Genomics Consortium schizophrenia case-control study (PGC_SCZ52_may13) (www.broadinstitute.org/mpg/ricopili/) at significance level $5.0 \times 10^{-8}$. Horizontal line: gene-wide significance threshold for association between 1566 VRK2 SNPS and VRK2 mRNA. This threshold was computed with the aid of matSpD ${ }^{33}$ taking into account the SNP correlations reflected by sample's LD-structure in the region of interest. The effective number $(n=174)$ of independent tests was computed using Li's procedure. TSS, transcription start site. 
longer form (Fig. 3). This suggests that it is one of the long isoforms of VRK2 that is involved in the aetiology of schizophrenia rather than one of the short isoforms. Future studies should aim to conclusively identify the exact transcript of VRK2 involved as well as confirm that the significant differences observed at the mRNA level are also present at the protein level.

In conclusion, our results give further support to the relevance of the VRK2 gene in schizophrenia susceptibility, and suggest that reduced gene expression might be involved in the underlying disorder mechanisms, potentially related to dysregulation of the immune system and impaired neurodevelopment. Moreover, we observed diagnostic specificity for schizophrenia (30\% explained variance $v$. healthy controls), with a distribution range which differs substantially from that of bipolar disorder and healthy controls (Fig. 2 and Fig. DS3). Such differences in the distributions encourage further studies to test the possibility of using VRK2 as a biomarker for schizophrenia.

\section{Martin Tesli, MD, PhD, Katrine Verena Wirgenes, MD, PhD, NORMENT, KG Jebsen Centre for Psychosis Research, Institute of Clinical Medicine, University of Oslo, Oslo, Division of Mental Health and Addiction, Oslo University Hospital, Oslo, Norway; Timothy Hughes, PhD, NORMENT, KG Jebsen Centre for Psychosis Research, Institute of Clinical Medicine, University of Oslo, Oslo, Division of Mental Health and Addiction, Oslo University Hospital, Oslo, Department of Medical Genetics, Oslo University Hospital, Oslo, Norway; Francesco Bettella, PhD, NORMENT, KG Jebsen Centre for Psychosis Research, Institute of Clinical Medicine, University of Oslo, Oslo, Division of Mental Health and Addiction, Oslo University Hospital, Oslo, Norway; Lavinia Athanasiu, PhD, NORMENT, KG Jebsen Centre for Psychosis Research, Institute of Clinical Medicine, University of Oslo, Oslo, Division of Mental Health and Addiction, Oslo University Hospital, Oslo, Department of Medical Genetics, Oslo University Hospital, Oslo, Norway; Eva S. Hoseth, MD, Mari Nerhus, MD, Trine v. Lagerberg, PhD, NORMENT, KG Jebsen Centre for Psychosis Research, Institute of Clinical Medicine, University of Oslo, Oslo, Division of Mental Health and Addiction, Oslo University Hospital, Oslo, Norway; Nils E. Steen, MD, PhD, NORMENT, KG Jebsen Centre for Psychosis Research, Institute of Clinical Medicine, University of Oslo, Oslo, Division of Mental Health and Addiction, Oslo University Hospital, Oslo, Drammen District Psychiatric Centre, Clinic of Mental Health and Addiction, Vestre Viken Hospital Trust, Drammen, Norway; Ingrid Agartz, MD, PhD, NORMENT, KG Jebsen Centre for Psychosis Research, Institute of Clinical Medicine, University of Oslo, Oslo, Division of Mental Health and Addiction, Oslo University Hospital, Oslo, Department of Psychiatric Research, Diakonhiemmet Hospital, Oslo, Norway: Ingrid Research, Institute of Clinical Medicine, University of Oslo, Oslo, Norway, Division of Mental Health and Addiction, Oslo University Hospital, Oslo, Norway; Srdjan Djurovic, PhD, NORMENT, KG Jebsen Centre for Psychosis Research, Department of Clinical Science, University of Bergen, Bergen, Department of Medical Genetics, Oslo University Hospital, Oslo, Norway; Ole Andreas Andreassen, MD, PhD, NORMENT, KG Jebsen Centre for Psychosis Research, Institute of Clinical Medicine, University of Oslo, Oslo, Division of Mental Health and Addiction, Oslo University Hospital, Oslo, Norway}

Correspondence: Ole A. Andreassen, MD, PhD, NORMENT, KG Jebsen Centre for Psychosis Research, Building 49, Oslo University Hospital, Ullevål, Kirkeveien 166, PO Box 4956 Nydalen, 0424 Oslo, Norway. Email: m.s.tesli@medisin.uio.no

First received 10 Dec 2014, final revision 24 Jun 2015, accepted 24 Sep 2015

\section{Acknowledgements}

We thank all study participants for their involvement in the study, and the health professionals who facilitated our work. We also thank Thomas D. Bjella for assistance with the database. The work was supported by the Research Council of Norway, the South-East Norway Regional Health Authority and KG Jebsen Foundation.

\section{References}

1 Lichtenstein P, Yip BH, Bjork C, Pawitan Y, Cannon TD, Sullivan PF, et al. Common genetic determinants of schizophrenia and bipolar disorder in Swedish families: a population-based study. Lancet 2009; 373: 234-9.

2 Sullivan PF, Kendler KS, Neale MC. Schizophrenia as a complex trait: evidence from a meta-analysis of twin studies. Arch Gen Psychiatry 2003; 60 1187-92.

3 Schizophrenia Working Group of the Psychiatric Genomics C. Biological insights from 108 schizophrenia-associated genetic loci. Nature 2014; 511 421-7.

4 Dieset I, Djurovic S, Tesli M, Hope S, Mattingsdal M, Michelsen A, et al. Upregulation of NOTCH4 gene expression in bipolar disorder. Am J Psychiatry 2012; 169: 1292-300.
5 Wirgenes KV, Sonderby IE, Haukvik UK, Mattingsdal M, Tesli M, Athanasiu L, et al. TCF4 sequence variants and mRNA levels are associated with neurodevelopmental characteristics in psychotic disorders. Trans/ Psychiatry 2012; 2: e112.

6 Wirgenes KV, Tesli M, Inderhaug E, Athanasiu L, Agartz I, Melle I, et al. ANK3 gene expression in bipolar disorder and schizophrenia. Br J Psychiatry 2014; 205: $244-5$

7 Li M, Wang Y, Zheng XB, Ikeda M, Iwata N, Luo XJ, et al. Meta-analysis and brain imaging data support the involvement of VRK2 (rs2312147) in schizophrenia susceptibility. Schizophr Res 2012; 142: 200-5.

8 Steinberg S, de Jong S, Irish Schizophrenia Genomics C, Andreassen OA Werge $T$, Borglum $A D$, et al. Common variants at VRK2 and TCF4 conferring risk of schizophrenia. Hum Mol Genet 2011; 20: 4076-81.

9 Kerner B, Rao AR, Christensen B, Dandekar S, Yourshaw M, Nelson SF. Rare genomic variants link bipolar disorder with anxiety disorders to creb-regulated intracellular signaling pathways. Front Psychiatry 2013; 4: 154.

10 Psychiatric GWAS Consortium Bipolar Disorder Working group. Large-scale genome-wide association analysis of bipolar disorder identifies a new susceptibility locus near ODZ4. Nature Genet 2011; 43: 977-83.

11 Nezu J, Oku A, Jones MH, Shimane M. Identification of two novel human putative serine/threonine kinases, VRK1 and VRK2, with structural similarity to vaccinia virus B1R kinase. Genomics 1997; 45: 327-31.

12 Monsalve DM, Merced T, Fernandez IF, Blanco S, Vazquez-Cedeira M, Lazo PA. Human VRK2 modulates apoptosis by interaction with $\mathrm{BCl}-\mathrm{XL}$ and regulation of BAX gene expression. Cell Death Dis 2013; 4: e513.

13 Blanco S, Sanz-Garcia M, Santos CR, Lazo PA. Modulation of interleukin-1 transcriptional response by the interaction between VRK2 and the JIP1 scaffold protein. PLOS One 2008; 3: e1660.

14 Sanz-Garcia M, Vazquez-Cedeira M, Kellerman $E$, Renbaum $P$, Levy-Lahad $E$, Lazo PA. Substrate profiling of human vaccinia-related kinases identifies coilin, a Cajal body nuclear protein, as a phosphorylation target with neurological implications. J Proteomics 2011; 75: 548-60.

15 Helbig I, Lowenstein DH. Genetics of the epilepsies: where are we and where are we going? Curr Opin Neurol 2013; 26: 179-85.

16 Andreassen OA, Harbo HF, Wang Y, Thompson WK, Schork AJ, Mattingsdal $\mathrm{M}$, et al. Genetic pleiotropy between multiple sclerosis and schizophrenia but not bipolar disorder: differential involvement of immune-related gene loci. Mol Psychiatry 2015; 20: 207-14.

17 Kendler KS, Walsh D. Schizophreniform disorder, delusional disorder and psychotic disorder not otherwise specified: clinical features, outcome and familial psychopathology. Acta Psychiatr Scand 1995; 91: 370-8.

18 Tesli M, Espeseth T, Bettella F, Mattingsdal M, Aas M, Melle I, et al. Polygenic risk score and the psychosis continuum model. Acta Psychiatr Scand 2014 130: $311-7$

19 Andreassen OA, Thompson WK, Schork AJ, Ripke S, Mattingsdal M, Kelsoe J, et al. Improved detection of common variants associated with schizophrenia and bipolar disorder using pleiotropy-informed conditional false discovery rate. PLOS Genet 2013; 9: e1003455.

20 International Schizophrenia Consortium. Common polygenic variation contributes to risk of schizophrenia and bipolar disorder. Nature 2009; 460 748-52.

21 Spitzer RL, Williams JB, Gibbon M, First MB. The Structured Clinical Interview for DSM-III-R (SCID). I: History, rationale, and description. Arch Gen Psychiatry 1992; 49: 624-9.

22 Athanasiu L, Mattingsdal M, Kahler AK, Brown A, Gustafsson O, Agartz I, et al. Gene variants associated with schizophrenia in a Norwegian genome-wide study are replicated in a large European cohort. J Psychiatr Res 2010; 44 $748-53$

23 Djurovic S, Gustafsson O, Mattingsdal M, Athanasiu L, Bjella T, Tesli M, et al. A genome-wide association study of bipolar disorder in Norwegian individuals, followed by replication in Icelandic sample. J Affect Disord 2010; 126: $312-6$

24 Hellemans J, Mortier G, De Paepe A, Speleman F, Vandesompele J. qBase relative quantification framework and software for management and automated analysis of real-time quantitative PCR data. Genome Biol 2007; 8: R19.

25 Purcell S, Neale B, Todd-Brown K, Thomas L, Ferreira MA, Bender D, et al. PLINK: a tool set for whole-genome association and population-based linkage analyses. Am J Hum Genet 2007; 81: 559-75.

26 Li Y, Willer CJ, Ding J, Scheet P, Abecasis GR. MaCH: using sequence and genotype data to estimate haplotypes and unobserved genotypes. Genet Epidemiol 2010; 34: 816-34.

27 Bohn MJ, Babor TF, Kranzler HR. The Alcohol Use Disorders Identification Test (AUDIT): validation of a screening instrument for use in medical settings. J Stud Alcohol 1995; 56: 423-32. 
28 Berman AH, Bergman H, Palmstierna T, Schlyter F. Evaluation of the Drug Use Disorders Identification Test (DUDIT) in criminal justice and detoxification settings and in a Swedish population sample. Eur Addict Res 2005; 11: 22-31.

29 Kay SR, Fiszbein A, Opler LA. The Positive and Negative Syndrome Scale (PANSS) for schizophrenia. Schizophr Bull 1987; 13: 261-76.

30 Rush AJ, Gullian CM, Basco MR, Jarrett RB, Trivedi MH. The Inventory of Depressive Symptomatology (IDS): psychometric properties. Psychol Med 1996; 26: 477-86.

31 Endicott J, Spitzer RL, Fleiss JL, Cohen J. The Global Assessment Scale. A procedure for measuring overall severity of psychiatric disturbance. Arch Gen Psychiatry 1976; 33: 766-71.

32 Young RC, Biggs JT, Ziegler VE, Meyer DA. A rating scale for mania: reliability, validity and sensitivity. Br J Psychiatry 1978; 133: 429-35.

$33 \mathrm{Li} \mathrm{J,} \mathrm{Ji} \mathrm{L.} \mathrm{Adjusting} \mathrm{multiple} \mathrm{testing} \mathrm{in} \mathrm{multilocus} \mathrm{analyses} \mathrm{using} \mathrm{the}$ eigenvalues of a correlation matrix. Heredity 2005; 95: 221-7.

34 Bergen SE, O'Dushlaine CT, Ripke S, Lee PH, Ruderfer DM, Akterin S, et al Genome-wide association study in a Swedish population yields support for greater CNV and $\mathrm{MHC}$ involvement in schizophrenia compared with bipolar disorder. Mol Psychiatry 2012; 17: 880-6.

35 Wickham H. ggplot2: Elegant Graphics for Data Analysis (1st edn). Springer 2009.

36 Epicure Consortium, EMINet Consortium. Genome-wide association analysis of genetic generalized epilepsies implicates susceptibility loci at 1q43, 2p16.1, 2q22.3 and 17q21.32. Hum Mol Genet 2012; 21: 5359-72.
37 Altamura AC, Pozzoli S, Fiorentini A, Dell'osso B. Neurodevelopment and inflammatory patterns in schizophrenia in relation to pathophysiology. Prog Neuropsychopharmacol Biol Psychiatry 2013; 42: 63-70.

38 Hope S, Ueland T, Steen NE, Dieset I, Lorentzen S, Berg AO, et al. Interleukin 1 receptor antagonist and soluble tumor necrosis factor receptor 1 are associated with general severity and psychotic symptoms in schizophrenia and bipolar disorder. Schizophr Res 2013; 145: 36-42.

39 Rimol LM, Hartberg CB, Nesvag R, Fennema-Notestine C, Hagler DJ Jr, Pung $\mathrm{CJ}$, et al. Cortical thickness and subcortical volumes in schizophrenia and bipolar disorder. Biol Psychiatry 2010; 68: 41-50.

40 Simonsen C, Sundet K, Vaskinn A, Birkenaes AB, Engh JA, Faerden A, et al. Neurocognitive dysfunction in bipolar and schizophrenia spectrum disorders depends on history of psychosis rather than diagnostic group. Schizophr Bull 2011; 37: 73-83.

41 Sullivan PF, Fan C, Perou CM. Evaluating the comparability of gene expression in blood and brain. Am J Med Genet B Neuropsychiatr Genet 2006; 141B: 261-8.

42 Takahashi M, Hayashi $\mathrm{H}$, Watanabe $\mathrm{Y}$, Sawamura K, Fukui N, Watanabe J, et al. Diagnostic classification of schizophrenia by neural network analysis of blood-based gene expression signatures. Schizophr Res 2010; 119: 210-8.

43 Wright FA, Sullivan PF, Brooks Al, Zou F, Sun W, Xia K, et al. Heritability and genomics of gene expression in peripheral blood. Nature Genet 2014; 46: $430-7$.

\section{psychiatry} and sacred texts

\section{The Qur'an, Chapter 93: The Morning Hours}

\author{
Ibtesham T. Hossain
}

The Qur'an is compiled of 114 chapters delving into a multitude of existential themes which provide the perfect ingredients for a human psyche - influencing the emotions, thoughts and behaviour of over a billion people worldwide.

Chapter 93 in combination with its context, is of particular interest from a psychiatric point of view because it provides a framework for overcoming depression. The opening verse begins with an oath, 'By the morning brightness' (verse 1) referring to the early part of the day as being a time of activity and full of life, directing a person's mind to positive thoughts. This is followed by 'And by the night when it grows still' (verse 2), implying that the stillness of the night provides calmness, the juxtaposition of the verses highlighting the contrasting emotions a person faces during the day and night. 'Your Lord has not forsaken you, nor does He hate you' (verse 3) - powerfully provides a sense of belonging and dispels the notion of helplessness.

The closing verses then describe how a person's situation can be changed regardless of their circumstances, inducing a sense of control through reliance and gratitude, all coming together to foster the take-home message - hope. 